\title{
Comunidades de aprendizaje: ¿qué aportan a los voluntarios y voluntarias universitarios?
}

\section{Learning communities: what is the university volunteers contribution?}

\author{
María del Mar PRADOS GALLARDO, María-Teresa GÓMEZ-DEL-CASTILLO y \\ Antonio AGUILERA-JIMÉNEZ \\ Universidad de Sevilla
}

Recibido: Octubre 2014

Evaluado: Marzo 2015

Aceptado: Abril 2015

\section{Resumen}

En este trabajo se presentan las valoraciones de las y los estudiantes universitarios que han participado como voluntarios en centros constituidos como Comunidad de Aprendizaje. Se ha realizado un análisis del contenido de 23 informes entregados por alumnos y alumnas de la titulación de Psicología de la Universidad de Sevilla. Los resultados se han agrupado en cinco bloques que hacen referencia a: a) para qué les ha servido colaborar en el voluntariado; b) cómo se han sentido durante esta colaboración; c) los aspectos positivos que quieren destacar de esta experiencia, d) los aspectos negativos de la misma y por último e) las propuestas de mejora que quieran formular. La principal conclusión de este trabajo es que, a juicio de los participantes, esta experiencia les ha aportado muchos beneficios a nivel profesional y personal, siendo una práctica formativa que valoran muy positivamente y que no sólo recomendarían a otros compañeros y compañeras sino que volverían a repetir.

Palabras clave: enseñanza universitaria, voluntariado, aprendizaje dialógico, aprendizaje servicio, interacción.

\begin{abstract}
This paper presents the assessment of the voluntary students who have participated in centers established as Learning Community. We have carried out a content analysis of 23 reports of students of the degree in psychology from the University of Sevilla. The results have been grouped into five blocks that refer to: a) what benefit have they obtained from collaborating in the voluntary service; b) how have they felt during the collaboration; c) what are the positive aspects that they would like to highlight from the experience; d) what are the negative aspects of the experience and, lastly; e) improvement proposals that they would like to put forward. The main conclusion of this paper is that, in the view of its participants, this experience has brought them many benefits both at professional and personal levels, being a highly positively valued practice that they would recommend to others colleagues and they would repeat.
\end{abstract}

Keywords: higher education, volunteering, dialogic learning, learning service, interaction. 
El fin último de este trabajo es hacer visible la valoración que hacen las y los estudiantes de la Universidad de Sevilla que han participado como voluntarios en Comunidades de Aprendizaje (de aquí en adelante CdA). En la última década están proliferando los estudios y artículos que nos muestran las CdA como generadoras de prácticas educativas exitosas en la superación del fracaso escolar (Jaussi y Luna, 2002), en la mejora del aprendizaje instrumental y la convivencia (Elboj, Puigdellívol, Soler y Valls, 2002; Flecha, 2009; Flecha, García y Rudd, 2011; entre otros), o incluso para la transformación social y educativa de un barrio (García y Villar, 2011), sin embargo, son muy escasos los trabajos que se centren en el papel del voluntariado en CdA (Prados y Aguilera, 2013a, b). En este sentido, podemos citar el trabajo de Vieira (2010) centrado en analizar el papel del voluntariado en CdA de cara a favorecer la inclusión, la mejora del aprendizaje, la convivencia y la aceptación de las diferencias dentro de la comunidad, o los trabajos de Prados y Aguilera que han destacado los beneficios que el proyecto $\mathrm{CdA}$ tiene de cara a los voluntarios y voluntarias universitarios (Conejo, Fernández, Nagy y Prados, 2013, Prados y Aguilera, 2013a, 2013b).

\section{¿Qué es una Comunidad de Aprendizaje?}

Las CdA disponen de una base sólida, que permite remontarnos en el plano internacional a prácticas de éxito como el School Development Program de la Yale University (Comer et al. 1999); el proyecto Success for All de la Johns Hopkins University (Slavin, 1996); Accelerated Schools de la Stanford University (Levin, 1987) $\mathrm{y}$, en especial, las actuaciones educativas de éxito identificadas en las escuelas europeas a través del proyecto INCLUD-ED (INCLUD-ED Consortium, 2011). Este proyecto ha sido una de las investigaciones de mayor envergadura que se han realizado en Europa sobre educación. Se ha desarrollado entre los años 2006 y 2011 por más de cien investigadores e investigadoras de catorce países europeos junto con profesorado de distintos niveles educativos y de diferentes regiones de nuestro continente. Fue el único proyecto integrado del VI Programa Marco de la Comisión Europea (2002-06), en la convocatoria del 2005 dedicado a la educación. Ha sido también el único proyecto integrado del área de Ciencias Sociales, Económicas y Humanas del VI Programa Marco, coordinado por un centro de investigación español: el CREA de la Universidad de Barcelona (Valls y Mulcahy, 2012).

El fin último de INCLUD-ED fue identificar, a partir del análisis de literatura científica, políticas educativas y prácticas de éxito, las estrategias educativas que contribuyen a superar las desigualdades y promueven la cohesión social, así como aquellas otras que generan mayor exclusión (Valls y Mulcahy, 2012). No podemos detenernos mucho más en describir el proyecto, para ello puede consultarse Valls, Prados y Aguilera (2014), pero si tenemos que resumir sus resultados, muy brevemente, podríamos decir que, las formas de agrupación del alumnado y la manera en que se gestionan los recursos humanos de un centro educativo se han revelado como dos excelentes indicadores de eficiencia y equidad, así como dos elementos clave para el éxito académico de niños y niñas. Justo, dos puntos que están presentes en las 
actuaciones educativas de éxito identificadas en el proyecto y que se implementan en los centros transformados en CdA.

Estos centros incorporan en su quehacer diario estas actuaciones educativas que la comunidad científica internacional ha identificado como las de mayor éxito en la superación del fracaso escolar y en la mejora de la convivencia en los centros educativos. Estas actuaciones afectan al quehacer diario de los centros educativos en todos sus aspectos, tanto en aquellas prácticas diarias relacionadas con los procesos de enseñanza y aprendizaje de toda la comunidad -formación de familiares, participación educativa de toda la comunidad, grupos interactivos, tertulias dialógicas, formación dialógica del profesorado y resolución dialógica de conflictos- como en la gestión democrática de los centros -mediante comisiones mixtas, asambleas con alumnos y alumnas y normas consensuadas por toda la comunidad educativa- (Aguilar, Alonso, Padrós y Pulido, 2010; Puigvert y Santacruz, 2006).

Uno de los elementos comunes a todas estas actuaciones de éxito es su fundamentación en una concepción dialógica del aprendizaje (Aubert, Flecha, García, Flecha, y Racionero, 2008; Aubert, García y Racionero, 2009; Flecha, 1997;). El aprendizaje dialógico es el que se produce en interacciones basadas en el diálogo igualitario, es decir, un diálogo en el que diferentes personas aportan argumentos en condiciones de igualdad, para llegar a consenso, partiendo de que quieren entenderse hablando desde pretensiones de validez (Elboj, et al. 2002).

Las CdA además de sustentarse en prácticas de éxito, se fundamentan en las teorías más referenciadas actualmente en las ciencias sociales como son, entre otras, las aportaciones de Vygotski (1979) y Mead (1973) desde la Psicología, de Freire (1970) desde la Pedagogía, o de Habermas (1987) y Beck (1998) desde la Sociología. Estos autores vienen a destacar la relevancia de las interacciones sociales para el aprendizaje y el desarrollo personal. Defienden que todas las interacciones que las personas mantienen en su día a día influyen en su proceso de aprendizaje. Como apuntaba Freire, todas las personas que forman parte del entorno del niño o niña influyen en su aprendizaje y por tanto deben planificarlo conjuntamente. Además, en estas interacciones la comunicación tiene una especial relevancia. Como afirmaba Habermas (1987) en la teoría de la acción comunicativa, todas las personas son capaces de realizar el acto de la comunicación y poseen las habilidades comunicativas básicas, entendiendo como habilidad comunicativa básica aquella característica que nos permite comunicarnos y actuar en nuestro entorno (Flecha y Puigvert, 2002).

\section{El papel del voluntariado en Comunidades de Aprendizaje}

Las prácticas educativas que se derivan de esta concepción comunicativa del aprendizaje exigen el incremento de las interacciones del alumnado entre sí y con otros agentes educativos distintos a su profesor o profesora habitual (familiares, otros docentes, voluntariado externo al centro, etc.). La Universidad de Sevilla está implicada en la formación de voluntariado universitario que colabora en los grupos interactivos, biblioteca tutorizada, tertulias dialógicas y otras prácticas comunicativas de los centros de la provincia de Sevilla que se han constituido como CdA. Así, desde 
el año 2007 la Universidad de Sevilla cuenta con una Actividad de Libre Configuración, que tras la implantación de las nuevas titulaciones de Grado se ha incluido en el Catálogo de Actividades Universitarias, Culturales, Deportivas, de Representación Estudiantil, Solidarias y de Cooperación, denominada "Prácticas de Intervención Psicoeducativa en Comunidades de Aprendizaje" hasta el curso 2013-14 $\mathrm{y}$, actualmente "Intervención Psicoeducativa en Comunidades de Aprendizaje" (Prados, López, Sánchez y Aguilera, 2011). La matrícula a esta actividad está abierta a estudiantes de cualquier titulación interesados por la educación, con prioridad para aquellos que procedan de titulaciones más afines (Grados de Maestro, Pedagogía, Psicopedagogía, Trabajo Social, Educación Social o Psicología). Hasta el curso académico 2012-13, una vez finalizada la experiencia, los estudiantes hacían entrega de un informe necesario para que la actividad le fuera reconocida como merecedora de los créditos por los que se computa. Se trataba de un documento de reflexión y revisión sobre la experiencia vivida cuyas características se detallan en el apartado de metodología.

La rica información que encontramos en estos informes llevó al profesorado de la asignatura a plantearse realizar un análisis más profundo de los mismos. Este análisis muestra un gran nivel de satisfacción por parte de los alumnos y alumnas universitarios que participan en esta experiencia (Prados y Aguilera, 2013a, b)

Es en este marco donde tiene cabida el trabajo que aquí se presenta cuyos objetivos son:

- Informar a la comunidad científica del punto de vista de los voluntarios y voluntarias sobre el proyecto Comunidades de Aprendizaje.

- Informar a la comunidad científica de los múltiples beneficios que aporta este proyecto, y la participación en sus prácticas de éxito, a los voluntarios y voluntarias que participan en las mismas.

- Dar voz a los estudiantes de Psicología que han sido voluntarios y voluntarias en Comunidades de Aprendizaje a partir de sus propias narraciones.

\section{Método}

Con los fines propuestos, se ha analizado el contenido de los informes entregados por el alumnado que ha participado en la experiencia.

\section{Participantes}

23 estudiantes universitarios de ambos sexos que cursan estudios de Psicología y que han desarrollado esta actividad en el marco de la materia de libre configuración y de reconocimiento académico "Prácticas de Intervención Psicoeducativa en Comunidades de Aprendizaje". En el desarrollo de la misma han sido voluntarios en dos de las CdA de la provincia de Sevilla en los cursos académicos 2010/2011 y $2011 / 2012$. 


\section{Instrumentos}

Se han analizado los informes entregados por los 23 alumnos y alumnas. Cada informe constaba de los siguientes puntos:

a. Descripción de las tareas realizadas y el contexto en el que se han realizado.

b. Valoración de esta experiencia (aspectos positivos y negativos) en función de una serie de criterios o expectativas previos y justificando el porqué de dicha valoración.

c. Desarrollar propuestas de actuación que sean coherentes con la valoración realizada. Estas propuestas pueden referirse tanto a la educación en general como a la experiencia particular de $C d A$.

\section{Procedimiento}

Para el análisis de los 23 informes se ha utilizado una metodología cualitativa basada en el análisis del contenido del discurso de los mismos. Siguiendo el contenido del discurso de las y los estudiantes en sus informes, el análisis final ha sido realizado a partir de las siguientes dimensiones: a) utilidad o funcionalidad que han encontrado a la realización del voluntariado, b) sentimientos asociados a la experiencia vivida en los centros, c) aspectos positivos, d) aspectos negativos, y e) propuestas de mejora. Además, estas dimensiones han sido cruzadas con las actividades en las que han participado como voluntarios y voluntarias: a) aportaciones sobre CdA en general, b) Grupos interactivos, c) Biblioteca autorizada, d) Tertulias literarias con adultos, e) Tertulias pedagógicas con adultos, f) otros.

El procedimiento seguido para llegar a estas dimensiones ha sido el siguiente:

1. Dos investigadores, de manera independiente, llevaron a cabo una primera lectura y categorización de las aportaciones encontradas en los informes atendiendo a los tres grandes apartados de los mismos: aspectos positivos, aspectos negativos y propuestas de mejora y, en función de las actividades en las que han participado como voluntarios y voluntarias (Grupos Interactivos, Biblioteca autorizada, etc.)

2. Se llevó a cabo una puesta en común del trabajo realizado y discusión y búsqueda de consenso ante los desacuerdos. Se encontró que era importante incluir dos dimensiones más a) utilidad o funcionalidad que han encontrado a la realización del voluntariado, y b) sentimientos asociados a la experiencia vivida en los centros.

3. Segunda lectura de los informes y elaboración de un primer listado de categorías emergentes.

4. Discusión en torno a las categorías, reelaboración y definición de las mismas. 
5. Codificación de los informes, por parte de dos investigadores, aplicando el sistema de categoría creado.

6. Comprobación de la fiabilidad intercodificadores.

7. Nueva discusión en torno a las categorías, reelaboración y definición de las mismas.

8. Codificación de todos los informes, por parte de dos investigadores, aplicando el sistema de categoría creado.

9. Comprobación de la fiabilidad intercodificadores.

El primer momento del análisis estuvo centrado en el contenido de las narrativas de los participantes. Éstas fueron leídas varias veces y las distintas aportaciones relativas a los aspectos positivos, negativos y propuestas de mejora identificadas fueron anotadas estableciéndose un listado de las mismas. A partir de dicho listado se elaboró un sistema de categorías emergentes que se ha ido probando, discutiendo y consensuando por todos los miembros del equipo de investigación (tres). Finalmente, dos investigadores categorizaron las aportaciones individualmente obteniéndose un acuerdo entre los mismos del $85 \%$. Recordemos que el índice de fiabilidad baja queda establecido en un coeficiente inferior al 70\% (Fox, 1981).

\section{Resultados}

Los resultados que aquí se presentan forman parte de un proyecto más amplio que tiene como objetivo general conocer la valoración que hacen de su experiencia el voluntariado universitario en CdA. Pese a la utilización de una metodología de corte cualitativo, centrada en el análisis del contenido de los informes, nos hemos permitido la licencia de hacer un somero análisis numérico. De cara a que se pueda tener una visión general de los datos, en la tabla I se presenta el número de aportaciones relativas a cada actuación de las desarrolladas dentro de las $\mathrm{CdA}$. 


\begin{tabular}{|c|c|c|c|c|c|c|c|}
\hline \multirow[b]{2}{*}{$\begin{array}{c}\text { Dimensiones } \\
\text { De análisis }\end{array}$} & \multicolumn{7}{|c|}{ Actuaciones educativas } \\
\hline & $\begin{array}{c}\text { Cuestiones } \\
\text { generales }\end{array}$ & $\begin{array}{c}\text { Grupos } \\
\text { interactivos }\end{array}$ & $\begin{array}{l}\text { Biblioteca } \\
\text { tut. }\end{array}$ & $\begin{array}{c}\text { Tertulias } \\
\text { literarias } \\
\text { con } \\
\text { adultos } \\
\end{array}$ & $\begin{array}{c}\text { Tertulias } \\
\text { pedagógicas } \\
\text { con adultos }\end{array}$ & Otros & Total \\
\hline $\begin{array}{c}\text { Para qué me } \\
\text { ha servido }\end{array}$ & 44 & 16 & 0 & 11 & 8 & 0 & $\begin{array}{c}75 \\
20.2 \%\end{array}$ \\
\hline $\begin{array}{c}\text { Cómo me he } \\
\text { sentido }\end{array}$ & 0 & 33 & 2 & 3 & 2 & 0 & $\begin{array}{c}40 \\
10.8 \% \\
\end{array}$ \\
\hline $\begin{array}{l}\text { Aspectos } \\
\text { positivos }\end{array}$ & 67 & 68 & 0 & 43 & 8 & 5 & $\begin{array}{c}191 \\
51.6 \% \\
\end{array}$ \\
\hline $\begin{array}{l}\text { Aspectos } \\
\text { negativos }\end{array}$ & 3 & 17 & 2 & 5 & 0 & 1 & $\begin{array}{c}28 \\
7.6 \%\end{array}$ \\
\hline $\begin{array}{l}\text { Propuesta } \\
\text { de mejora }\end{array}$ & 19 & 7 & 3 & 2 & 1 & 4 & $\begin{array}{c}36 \\
9.7 \%\end{array}$ \\
\hline Total & $\begin{array}{c}133 \\
(39.75 \%)\end{array}$ & $\begin{array}{c}141 \\
(35.25 \%)\end{array}$ & $\begin{array}{c}7 \\
(1.75 \%)\end{array}$ & $\begin{array}{c}64 \\
(16.00 \%)\end{array}$ & $\begin{array}{c}19 \\
(4.75 \%)\end{array}$ & $\begin{array}{c}10 \\
(2.50 \\
\%) \\
\end{array}$ & $\begin{array}{c}374 \\
(100 \% \\
)\end{array}$ \\
\hline
\end{tabular}

Tabla I: Número de aportaciones recogidas en cada dimensión y actuación

Este trabajo se centrará en las aportaciones de los y las estudiantes referidas a aspectos generales del proyecto Comunidades de Aprendizaje. Es decir, los datos recogidos en la primera columna.

\begin{tabular}{|c|c|c|}
\hline DIMENSIONES & \multicolumn{2}{|c|}{$\begin{array}{l}\mathbf{N}(\%) \text { de cuestiones } \\
\text { generales }\end{array}$} \\
\hline ¿Para qué me ha servido? & $44(33.1 \%)$ & \multirow{3}{*}{$\begin{array}{c}111 \\
(83.5 \%)\end{array}$} \\
\hline ¿Cómo me he sentido? & 0 & \\
\hline Aspectos positivos & $67(50.4 \%)$ & \\
\hline Aspectos negativos & $3(2.2 \%)$ & \multirow{2}{*}{$22(16.5 \%)$} \\
\hline Propuesta de mejora & $19(14.3 \%)$ & \\
\hline TOTAL & 133 & $\%)$ \\
\hline
\end{tabular}

Tabla II: Análisis cuantitativo de las aportaciones por dimensiones dentro de la actuación cuestiones generales

Como puede apreciarse en la tabla II un primer análisis numérico muestra que la mayoría de las aportaciones enmarcadas dentro de la columna "cuestiones generales" $(83.5 \%)$ corresponden a las dimensiones que hablan favorablemente de CdA. Esto es la dimensión "¿Para qué me ha servido" (33.1\%) y "Aspectos positivos” (50.4\%). En el 
otro extremo se podrían situar las dimensiones que recogen aportaciones que se refieren de forma no positiva a las CdA. Éstas suman algo menos del $17 \%$ de las aportaciones siendo tan sólo el $2.2 \%$ las que se refieren a "Aspectos negativos" y $14.3 \%$ las que recogen "Propuestas de mejora", muchas de las cuales, como se verá más adelante, no se refieren a mejorar aspectos considerados negativos sino a avanzar en los valorados positivamente (por ejemplo, "Extender Comunidades de Aprendizaje a otros centros" o "Incrementar la presencia de Comunidades de Aprendizaje en la formación universitaria").

A continuación se presentan extractos que ejemplifican las aportaciones identificadas en las narrativas del alumnado en función de la categorización que se ha realizado de las mismas. Es decir, para cada una de las dimensiones que se presentan en las tablas I y II, que son resultado del proceso de análisis de las narrativas, se mostrarán algunos ejemplos.

\section{¿Para qué me ha servido el voluntariado en Comunidades de Aprendizaje?}

Como puede apreciarse en la tabla II, esta dimensión es la que cuenta con más enunciados categorizados en la misma. En concreto, 44 afirmaciones (33.1\%) de las 133 identificadas en los informes en relación con cuestiones generales de las CdA. Atendiendo a las mismas más detenidamente, pueden clasificarse en dos grandes tipos. Así, podría decirse que las principales aportaciones que el voluntariado ha tenido para el alumnado que en él han participado ha sido su contribución a nivel personal y social. Algunas afirmaciones como "Ha sido una de las experiencias que más me han llenado, tanto como persona como profesionalmente" o "Ha sido una de las actividades más enriquecedora en mi vida como estudiante y como persona" son muy generales. Sin embargo, otros alumnos y alumnas son mucho más explícitos en sus comentarios respecto a lo que las CdA les han aportado.

\section{a) Contribución de las $\mathrm{CdA}$ a nivel personal}

En cuanto a las aportaciones a nivel personal pueden encontrarse en los informes afirmaciones que hacen referencia a que gracias a esta experiencia las personas voluntarias han madurado, superado prejuicios, desarrollado valores o incluso han tomado conciencia de las desigualdades sociales.

"Las características del entorno urbano del CEIP no son las más adecuadas para una escuela ni las que estamos acostumbrados a ver, pero a veces es importante conocer aquello que no sabemos que existe. Para mí ha significado mucho saber que hay lugares así a tan solo unos metros de colegios en los que los niños salen a la calle y no tienen el peligro en ella. A veces no somos capaces de hacernos a la idea de cómo está el mundo de mal repartido y he descubierto esa sensación de estar haciendo algo bueno por un entorno que realmente lo necesita" 
"Para mí ha sido una actividad muy enriquecedora porque el hecho, tanto de acudir al colegio como ir a las tertulias, me ha servido para conocer otra cultura como la cultura gitana, desmitificar algunos prejuicios que tenía en relación con la cultura gitana y descubrir la importancia de conocer los valores y las características de una cultura para poder trabajar con ella"

\section{b) Contribución de las $\mathrm{CdA}$ a nivel profesional}

Centrándonos en las contribuciones que a nivel profesional se mencionan en los informes de los alumnos y alumnas voluntarios se encuentran que el hecho de haber participado en esta experiencia ha sido de utilidad para mejorar sus habilidades sociales y comunicativas, conocer nuevas metodologías docentes o, como una experiencia laboral en sí misma.

"De cara a mi futuro profesional empezamos a tratar con diferentes personas $y$ ver cómo se relacionan entre ellas, tanto escolares como docentes. Vemos también cómo van evolucionando los niños según la marcha de la clase, cómo cambian y cómo se acostumbran a una nueva forma de enseñanza"

"Mis habilidades sociales se han visto favorecidas a partir de esta experiencia. Tener que hablar para toda una clase, dirigir y controlar un grupo de trabajo, hablar con los profesores sobre el rendimiento de un alumno, etc. Me ha hecho obtener más desenvoltura en mi relación con los demás y, también, al hablar delante de muchas personas. Esto va ser muy enriquecedor para mi futuro profesional"

Especial relevancia e interés tienen dentro de esta categoría aquellas aportaciones que aluden expresamente a cómo el hecho de haber sido voluntario o voluntaria en una CdA lleva a conocer y comprender mejor este proyecto y todo lo que conlleva.

"He comprobado que todo el mundo es útil para ayudar, y que no es necesario tener estudios para ayudar a quien lo necesita. Creo que yo misma he aprendido más de los niños que los niños de mi"

"He conocido de primera mano las funcionalidades prácticas del aprendizaje dialógico, perspectiva teórico educativa desde la que me siento muy atraído y en consonancia con sus principales postulados"

\section{Aspectos positivos de Comunidades de Aprendizaje}

Como se aprecia en la tabla II, más de la mitad de los enunciados $(50.4 \%)$ se encuentran categorizados en esta dimensión que, al igual que la anterior, se ha dividido en dos grandes grupos. De un lado se encuentran aportaciones que destacan aspectos positivos de las CdA relacionados con las y los estudiantes de la Universidad y, de otro lado aportaciones que hacen referencia a aspectos positivos para las otras personas participantes y distintos agentes implicados en las mismas. 
a) Aspectos positivos de las CdA para las y los voluntarios de la Universidad

Algunos de los enunciados identificados en los informes en relación con esta dimensión son bastante generales y se limitan a decir que la experiencia ha sido positiva, enriquecedora, maravillosa, fantástica, bonita, inolvidable o satisfactoria pero sin entrar en matices. Ejemplo de ellos son:

"Mi experiencia en el proyecto de Comunidades de Aprendizaje ha sido, sin duda alguna, una de las más bonitas que he tenido en mi vida universitaria”"

"No he podido extraer ningún aspecto negativo. Nada de lo vivido y experimentado en estos meses en que he participado de voluntario ha repercutido en mí de forma negativa, ni a nivel personal ni tampoco a nivel profesional. Todo lo contrario: me ha enriquecido en todos los aspectos"

Sin embargo, encontramos otros enunciados que denotan lo positivo de las CdA haciendo referencia a aspectos más concretos como que recomendarían esta experiencia a otras personas:

"Mi valoración general de la experiencia es muy positiva, recomendando que se colabore si se tiene la posibilidad de hacerlo. Y apreciando las comunidades de aprendizaje como un método de enorme valor educativo y social no solo para el alumnado sino para todas aquellas personas que participen en las mismas"

"No tengo dudas de que he recibido más de lo que he podido aportar ya que la experiencia ha sido mejor de lo que esperaba. Animo a todo aquel que tenga dudas sobre participar el año que viene; que no se lo piense un segundo"

O que repetirían la experiencia:

"Posiblemente el año que viene siga de voluntaria en el colegio y siga participando y formando parte de Comunidades de Aprendizaje”

"Es muy probable que el curso que viene realice un máster en Barcelona y me gustaría seguir allí asistiendo como voluntaria a algún centro de Comunidades de Aprendizaje. Ya he visto algunos centros de Barcelona a los que podría asistir (...) desde el momento en que sepa con certeza que estaré en esa ciudad comenzaré a ponerme en contacto con ellos",

Cuando el voluntariado entra en concreciones, lo que más destaca es el buen trato recibido por parte del personal y de los alumnos y alumnas de los centros a los que han asistido. Del profesorado destacan aspectos como su calidad humana y lo que han podido aprender de ellos, así como la manera en que han sido acogidos e integrados como parte del centro hasta el punto de sentir que se confía en ellos y que sus opiniones son tenidas en cuenta. Algunos ejemplos son: 
"He de nombrar la calidad humana recibida del profesorado. No solo me han mostrado la metodología, sino también el camino para ser un buen educador a través de la comprensión global del alumnado y sus problemas"

"A la tutora tengo que agradecerle que ha sido una persona que me integró como una más en el aula, contando conmigo para excursiones, debates e incluso en la integración con los demás maestros"

"El trato que he recibido por parte del profesorado del centro, notando cómo te valoran y cuentan con tu opinión, algo que no llegaba a esperar por entrar en el centro simplemente como voluntaria"

b) Aspectos positivos de las CdA para otros agentes

Se enmarcan aquí aquellos enunciados que hacen referencia a las buenas relaciones y trato observado en el día a día de en los centros educativos, no sólo hacia los voluntarios y voluntarias sino entre el personal docente, las familias y los niños y niñas. Destacan cómo positivo la presencia de familiares en el centro educativo y su implicación en el quehacer diario del mismo o; subrayan otros aspectos asociados a los principios de las CdA como que fomentan la cooperación frente al individualismo, desarrollan valores encaminados hacia la igualdad social y educativa o la visión de la diversidad cultural como una riqueza y no un inconveniente. Algunos ejemplos representativos de lo que se viene afirmando son los siguientes.

"Esa sensación de colaboración se aprecia entre los adultos, pero gracias a esas relaciones tan estrechas que tienen los adultos, los niños tienen también ese entusiasmo por ayudar al compañero"

"Otro aspecto que me gustaría destacar es la implicación que tienen los padres en el colegio, participando en los grupos interactivos, en las tertulias, en las actividades que organiza el centro, ... dando un ejemplo a seguir de la preocupación que deben tener los padres por la educación de sus hijos y no tomando el clásico papel de crítica a los profesores, sino tomando un papel activo en la educación de sus hijos e hijas"

"Otro aspecto positivo a destacar es la riqueza cultural que hay en el centro al tener matriculados a niños de diversas nacionalidades. Pero no es el simple hecho de tener estos alumnos en el centro, sino cómo aprovecha el colegio la presencia de ellos y, mediante actividades juegos, tradiciones, rutinas, etc. Hacen que cada niño conozca las características de las culturas de sus compañeros. A través de esta práctica es como han conseguido el clima de igualdad y respeto a la diversidad que hay en el centro"

\section{Aspectos negativos de Comunidades de Aprendizaje}

Como se podía apreciar en la tabla II los aspectos negativos se corresponden con 3 únicas afirmaciones recogidas, en este caso, por 3 alumnos diferentes. Dos de estos 
alumnos en sus informes hacen referencia a expectativas no cumplidas. En concreto consideran que la colaboración en CdA es más útil para estudiantes de magisterio que para los de psicología, pues esperaban haber realizado tareas más propias de profesionales de la psicología.

"Esperaba aprender algo más del trabajo práctico, desde el punto de vista psicológico y, bajo mi punto de vista, entiendo que esta asignatura es más útil a nivel formativo para estudiantes de magisterio"

"En cuanto a los puntos negativos yo destaco el hecho de que a los alumnos de psicología no se les permite trabajar en lo que más le podrían interesar, es decir, su tarea trata prácticamente del rol de profesor y no de psicólogo/a. Por ejemplo, en mi caso trabajé con los chicos de $5^{\circ}$ y $6^{\circ}$ en la asignatura de inglés y ello me suponía una dificultad ya que mi nivel de inglés, además de no ser muy bueno, no me motivaba. Lo que me hubiese gustado es hablar con los niños/as, profundizar en los temas que más les preocupan, desarrollar con ellos estrategias para una mayor integración e igualdad entre todos, así como competencias y habilidades sociales óptimas, además de trabajar los temas de diversidad cultural y de género, siempre llevados a cabo de forma adecuada a la edad del alumnado. Además sería también muy interesante que los voluntarios también pudieran ofrecer apoyo a los familiares, otra manera de hacerles saber que su opinión importa y de especial relevancia en la educación de sus hijos/as"

El otro de los aspectos negativos identificado hace referencia a que, para uno de los alumnos voluntarios, las $\mathrm{CdA}$ exigen mucho al profesorado.

"Requiere una mayor implicación por parte de los profesores que, si no están motivados o vocacionados, no trabajarán de la misma manera"

\section{Propuestas de mejora}

Las propuestas de mejora encontradas en los informes suponen un $14.3 \%$ del total de enunciados categorizados en "aspectos generales de las CdA". No obstante, como señalábamos anteriormente, no todas las propuestas de mejora que se hacen tienen relación con aspectos negativos de las $\mathrm{CdA}$ o de la participación del voluntariado universitario en ellas, sino que, por el contrario, algunas se derivan de la alta valoración que se hace de esta propuesta de transformación educativa y social. Es el caso de las propuestas encaminadas a la difusión de las $\mathrm{CdA}$ y de las prácticas educativas que en ellas se implementan a otros centros escolares.

a) Propuestas de mejora relativas a la divulgación y difusión de las $\mathrm{CdA}$ "Considero que todos los colegios, tanto públicos como privados, deberían tener la opción de contar con voluntarios que ayuden en todo lo que sea 
necesario para que se produzca una interacción ten enriquecedora como la que surge en los colegios que forman parte de Comunidades de Aprendizaje. Aunque lo perfecto es que todos los colegios formaran parte de Comunidades de Aprendizaje"

"Vería necesario una divulgación de la información tanto por los centros universitarios como entre toda la población, para de este modo aumentar la conciencia de todos sobre lo que se hace con este proyecto y que tengan presente que todos podemos ayudar y contribuir. Con la expansión de la información también se podría aumentar el número de colegios que se convirtiesen en Comunidades de Aprendizaje, sensibilizando al profesorado de dichos colegios y mostrándoles los excelentes resultados que dichas prácticas están teniendo en los colegios en los que se llevan a cabo. Con el aumento de las Comunidades de Aprendizaje habría más niños que se beneficiasen de los beneficios que éstas conllevan, como por ejemplo el hecho de que todos podemos ayudar y que la educación está en manos de todos, siendo todos iguales. Para la divulgación de la información se podrían hacer folletos en los que se explicara qué son y que hacen las comunidades de aprendizaje. Los podrían realizar los propios voluntarios donde dijeran lo que comunidades de aprendizaje les está aportando a ellos como personas. Estos folletos se repartirían por todas partes para informar a todas las personas. También se podrían usar los medios de comunicación como la radio o la tele, para divulgar estas ideas donde los voluntarios cuenten sus experiencias y de ese modo sensibilizar a la población"

Aparte de este tipo de propuestas, encontramos otros dos grandes bloques o tipos de aportaciones a este respecto. De un lado, aquellas propuestas que se refieren a aspectos directamente relacionados con la figura de las personas voluntarias en las $\mathrm{CdA} y$, de otro lado, las que recogen propuestas concretas de mejora de las CdA.

b) Propuestas de mejora relativas a las y los voluntarios de la Universidad

Se encuentran aquí aportaciones que señalan que debería informarse más de las CdA en la Universidad en dos sentidos, tanto mejorar la información al alumnado universitario sobre las posibilidades de colaborar en el voluntariado que se lleva a cabo en éstas como incluir dentro de los contenidos curriculares de la formación que se imparte en las Facultades de Psicología y de Educación el proyecto CdA. Ejemplo de ello son:

"Facilitar a los alumnos de la facultad de psicología la información necesaria para poder participar como personal de apoyo en Comunidades de Aprendizaje como, p.e., informando por correo-e"

"Pienso que sería interesante incorporar a la formación de los futuros docentes las dinámicas y principios teóricos y metodológicos que sustentan los modelos educativos propuestos desde Comunidades de Aprendizaje ya que, 
uno de los principales problemas que se encuentran a la hora de poner en marcha dichas comunidades, es la necesidad de formación requerida por parte del profesorado, lo cual se entiende por parte de algunos equipos directivos de centros y de organismos oficiales como una "pérdida de tiempo" y de "recursos"

Mención aparte merecen otras propuestas que se centran en la labor del voluntariado en los centros. Así, se propone que las personas voluntarias participen del diseño o planteamiento de actividades o que se cuente con estas personas de cara a la formación de familiares. Los siguientes fragmentos ejemplifican estas ideas.

"Aunque los profesores nos dijesen que podíamos proponer actividades para que los niños las realizasen, comentar que en nuestro caso en particular no propusimos ninguna por diversos motivos; por ello propongo que, para otros años, un requisito indispensable en estas prácticas sea que los voluntarios propongan algunas para que asi la participación sea mayor aún”

"Que los voluntarios se comprometiesen a realizar algunos talleres por la tarde destinados a padres y madres como: a) iniciación al uso de ordenadores $e$ internet; b)nutrición y salud alimentaria; c)estilos educativos familiares democrático, autoritario y permisivo; d) habilidades sociales, competencias comunicativas y negociación en conflictos; e) medios de comunicación, ..."

c) Propuestas concretas de mejora de las CdA

Resultan muy interesantes las propuestas identificadas de cara a la mejora de las CdA. Éstas están encaminadas hacia 1) la necesidad de aumentar la presencia de las familias en los centros y mejorar su formación; 2) impulsar la motivación y formación del profesorado o; 3) se centran en necesidades específicas de los niños o niñas como puede ser la necesidad de aprender estrategias de resolución de problemas interpersonales o mejorar sus habilidades sociales. Ejemplos de fragmentos de los informes que hacen referencia a cada uno de estos aspectos son:

En relación a los familiares:

"Es importante que los padres tuviesen la oportunidad de conocer las principales pautas educativas más acertadas y la adquisición de los recursos necesarios para conseguir una actuación lo más democrática posible. (...) Hay que elaborar actividades y talleres de este tipo en los colegios: sobre los distintos estilos educativos, técnicas y estrategias de educación, principios básicos del aprendizaje,..."

"En los propios colegios que son Comunidades de Aprendizaje habría que aumentar la participación de los padres de los niños. Para esto se podrían hacer reuniones donde se convocaran a todos los padres y que en ellas, los padres que participan en el colegio les cuenten sus experiencias y las cosas positivas que están sacando de dicha experiencia. Hablar tanto de los grupos 
interactivos como de las tertulias en las que hay pocos padres aún y que, además de tener la posibilidad de leer y debatir lo que leemos, también conocemos a personas, pasamos un buen rato y nos liberamos de las tensiones de la vida diaria"

En relación con el profesorado:

"Trataría de elaborar un plan para motivar al profesorado y 'vocacionarle', de cara al inicio de curso. Desarrollaría charlas con grupos de profesores para que comentasen sus preocupaciones, sus miedos, los posibles problemas que se les pudiesen plantear en clase y haría extensibles estas reuniones durante todo el curso, al menos una vez al mes. Plantearía la creación de un diario para cada profesor para que escribiesen cómo se sienten, o si se les ha ocurrido alguna actividad o tienen algún tipo de duda. Daría un cursillo por trimestre sobre cultura de la etnia gitana y cómo trabajar las habilidades sociales en niños y padres y habilitaría un banco de recursos, tanto de actividades como de ejercicios, etc. para que los profesores se ayudasen mutuamente. También establecería tutorías de profesorado veterano para que enseñen el ritmo de trabajo a los profesores recién llegados. Finalmente fecharía al menos dos comidas a lo largo del curso, una al inicio y otra al fin, para fomentar la cohesión entre los compañeros de trabajo"

En cuanto a los niños y niñas:

"Yo propondría que los voluntarios de psicología se embarcaran en realización de actividades que fomentaran la "resolución de problemas interpersonales así como la competencia social en los niños". Para el futuro de estos chicos es importante las competencias sociales, las habilidades comunicativas y de negociación y las estrategias de afrontamiento al stress o ante situaciones que provocan frustración. También estrategias más colaborativas y empáticas"

"Hay niños de cuarto de primaria que necesitan mayor refuerzo que el resto. Por ello propongo que ellos reciban este refuerzo con un profesor de apoyo y que sus padres se involucren en ayudarlos a seguir el mismo ritmo que el resto de sus compañeros"

\section{Discusión}

Podría decirse que la voz del alumnado de la Universidad de Sevilla que ha participado como voluntario en las dos CdA más antiguas de la provincia tiene mucho positivo que decir acerca de este proyecto. Los informes que se han analizado, elaborados por 23 estudiantes de psicología que han cursado la actividad de libre reconocimiento de créditos "Prácticas de Intervención Psicoeducativa en Comunidades de Aprendizaje" durante los cursos académicos 2010-11 y 2011-12, denotan que, a 
juicio de estos voluntarios y voluntarias, no sólo los niños y niñas que están en centros constituidos como CdA se benefician de las prácticas educativas que en ellas se realizan, sino que el voluntariado también lo hace y en gran medida.

Acorde con trabajos como el de Elboj y Niemala, (2010); Elboj, et al. (2002), Flecha, (2009), Includ-ed Consortium, (2011) o Valls y Kyriakides, (2013), entre otros, esta investigación apunta a que, desde el punto de vista del voluntariado universitario, las CdA están implicadas no sólo en la mejora del aprendizaje instrumental y la convivencia, sino también en la transformación social y educativa del centro y el barrio en el que este se encuentra (García y Villar, 2011). A lo largo del apartado de resultados se han podido leer fragmentos de los informes del voluntariado en los que se destacan muchas de las bondades de las CdA en este sentido: las buenas relaciones entre el profesorado y entre estos y el resto de la comunidad, así como el buen clima que se respira en las aulas, el fomento de valores encaminados hacia la igualdad social y educativa o la implicación familiar en la formación de sus hijos y en el día a día de la escuela.

Sin embargo, quizás lo más interesante que aporta este trabajo sea los beneficios que, a juicio del voluntariado universitario, tienen las CdA para ellos mismos. Así, como se ha visto en los resultados, éstos refieren que su participación en este proyecto ha supuesto importantes aportaciones tanto para su formación académica y profesional como para su desarrollo personal. Sus palabras hacen ver que han podido poner en práctica y contextualizar muchos de los aprendizajes llevados a cabo en su formación universitaria así como adquirir y construir nuevos aprendizajes. En este sentido, entendemos que esta asignatura lleva al alumnado que la cursa a realizar un aprendizaje servicio (Stanton, 1990), pues vincula estrechamente servicio y aprendizaje en una sola actividad educativa coordinada y coherente (Puig y Palos, 2006) consiguiendo que a través de ella se desarrollen diferentes competencias fundamentales como son el trabajo en equipo interdisciplinar, las habilidades en las relaciones personales o el compromiso ético (ANECA, 2005). Trabajos como el de Campo (2010), Francisco y Moliner (2010) o Tejada (2013) apuntan al aprendizaje servicio como una herramienta en el Marco Europeo de Educación Superior, promoviendo una de las funciones básicas de la Universidad junto con la formación para el trabajo, la formación para una ciudadanía activa. Es decir, la formación de ciudadanos críticos, activos y responsables con su entorno (Einfeld y Collins, 2008).

Finalmente, especial relevancia tiene el hecho de que los propios estudiantes demanden que experiencias como las que se llevan a cabo en torno a las CdA y las actuaciones educativas de éxito que en ellas se implementan, sean contenidos que se trabajen en la formación de los futuros profesionales de la educación. 


\section{Referencias bibliográficas}

AGENCIA NACIONAL DE EVALUACIÓN DE LA CALIDAD Y ACREDITACIÓN (2005). Libro blanco para el título de grado en magisterio. Madrid: ANECA.

AGUILAR, C., AlONSO, M. J., PADRÓS, M. Y PULIDO, M. A. (2010). Lectura dialógica y transformación en las Comunidades de Aprendizaje. Revista Interuniversitaria de Formación del Profesorado. 67(24, 1), 31-44.

AUBERT, A., FLECHA, A., GARCÍA, C., FLECHA, R., Y RACIONERO, S. (2008). Aprendizaje dialógico en la Sociedad de la Información. Barcelona: Hipatia.

AUBERT, A., GARCIA, C. Y RACIONERO, S. (2009). El aprendizaje dialógico. Cultura y Educación, 21(2), 129-139.

BECK, U. (1998). La sociedad del riesgo: hacia una nueva modernidad. Barcelona: Paidós.

CAMPO, L. (2010). El aprendizaje servicio en la Universidad. En M. Martínez (Ed.). Aprendizaje servicio y responsabilidad social en las universidades (pp. 81-91). Barcelona: Ediciones Octaedro, ICE UB.

COMER, J. P. et al. (ed.) (1999). Child by Child. The Comer process for change in Education. Nueva York: Teachers College Press.

CONEJO, E., FERNÁNDEZ, R., NAGY, C. Y PRADOS, M. M. (2013). Los alumnos y alumnas del grado de educación primaria como voluntarios en comunidades de aprendizaje. Comunicación presentada a las III Jornadas de Innovación Docente y Educativa: Respuesta en tiempos de Incertidumbre. Facultad de Ciencias de la Educación. Mayo de 2013. Sevilla.

EINFELD, A., Y COLLINS, D. (2008). "The Relationships Between ServiceLearning, Social Justice, Multicultural Competence, and Civic Engagement". Journal of College Student Development, 49(2), 95-109.

ELBOJ, C., Y NIEMALA, R. (2010). Sub-Communities of Mutual Learners in the Classroom: The case of Interactive Groups. Revista de Psicodidáctica, 15(2), 177189.

ELBOJ, C., PUIGDELlíVOL, I, SOLER, M. Y VALLS, R. (2002). Comunidades de aprendizaje: transformar la educación. Barcelona: GRAÓ.

FLECHA, R. (1997). Compartiendo palabras El aprendizaje de las personas adultas a través del diálogo. Barcelona: Paidós

FLECHA, R. (2009). Cambio, inclusión y calidad en las comunidades de aprendizaje. Cultura y Educación, 21(2), 157-159.

FLECHA, A., GARCÍA, R., Y RUDD, R. (2011). Using Health Literacy in School to Overcome Inequalities. European Journal of Education, 46(2), 209-218.

FLECHA, R. Y PUIGVERT, L. (2002). Las comunidades de aprendizaje: una apuesta por la igualdad educativa. REXE Revista de estudios y experiencias en educación. 
Concepción, Chile: Facultad de Educación de la Universidad Católica de la Santísima Concepción, 1(1), 11-20.

FRANCISCO, A. Y MOLINER, L. (2010). El Aprendizaje Servicio en la Universidad: una estrategia en la formación de ciudadanía crítica. REIFOP, 13 (4), 69-97. Recuperado de http://www.aufop.com -

FREIRE, P. (1970). Pedagogía del Oprimido. México: Siglo XXI

GARCÍA, J. Y VILLAR, C. (2011). La aportación del proyecto de comunidades de aprendizaje a la transformación social y educativa de un barrio. Tendencias pedagógicas, 18, 208-211

HABERMAS, J. (1987). Teoría de la acción comunicativa. Vol. I: Racionalidad de la acción y racionalización social. Vol. II: Crítica de la razón funcionalista. Madrid: Taurus.

INCLUD-ED Consortium (2011). Actuaciones de éxito en las escuelas europeas. Madrid: MEC. Recuperado de http://www.nesetweb.eu/sites/default/files/ actuaciones-de-exito-en-las-escuelas-europeas.pdf

JAUSSI, M. L. Y LUNA, F. (2002). Experiencias de éxito. Cuadernos de Pedagogía, 316 (Tema del Mes, septiembre), 39- 67.

LEVIN, H. M. (1987). Accelerated schools for the disadvantaged students. Educational Leadership, 44(6), 19-21.

MEAD, H. (1973). Espíritu, persona y sociedad. Barcelona: Paidós.

PRADOS, M. M., LÓPEZ, M., SÁNCHEZ, V. Y AGUILERA, A. (2011). Prácticas de Intervención Psicoeducativas en Comunidades de Aprendizaje. En Aguilera, A. y Gómez de Terreros, M. (Coord.). Actividad docente en el marco del espacio europeo de educación superior (pp.20-35). Sevilla: Instituto de Ciencias de la educación.

PRADOS, M. M. Y AGUILERA, A. (2013a). Los alumnos y alumnas de la Universidad de Sevilla como voluntarios en Comunidades de Aprendizaje. Andalucía educativa, Septiembre. Recuperado de http://www.juntadeandalucia.es/educacion/webportal/web/revista-andaluciaeducativa/en-portada/-/noticia/detalle/los-alumnos-y-alumnas-de-la-universidad-desevilla-como-voluntarios-en-comunidades-de-aprendizaje-1

PRADOS, M. M. Y AGUILERA, A. (2013b). Prácticas y voluntariado en Comunidades de Aprendizaje. Los alumnos universitarios tienen mucho que aportar a la comunidad educativa. Comunicación presentada al Segundo Congreso Internacional Multidisciplinar de Investigación Educativa (CIMIE). Tarragona, julio de 2013.

PUIG, J. M. Y PALOS, J. (2006). Rasgos pedagógicos del aprendizaje-servicio. Cuadernos de Pedagogía, 357, 60-63. 
PUIGVERT, L., Y SANTACRUZ, I. (2006). La transformación de centros educativos en comunidades de aprendizaje. Calidad para todas y todos. Revista de Educación, 339, 169-176.

SLAVIN, R.E. (1996). Education for all. Exton: Swets y Zeitlinger Publishers.

STANTON, T. (1990). Service Learning: Groping toward a definition. En kendall et al. Combinig Service and Learning: A resource Book for Community and Public Service Vol. 1, 65-67. Raleigh, North Carolina: National Society for Internships \& Experiential Education.

TEJADA FERNÁNDEZ, J. (2013). La formación de las competencias profesionales a través del aprendizaje servicio. Cultura y educación 25(3), 285-294.

VALLS, R., Y KYRIAKIDES, L. (2013): The power of Interactive Groups: how diversity of adults volunteering in classroom groups can promote inclusion and success for children of vulnerable minority ethnic populations. Cambridge Journal of Education, 43(1), 17-31.

VALLS, R. Y MULCAHY, C. (2012). El proyecto Includ-ed. Cuadernos de Pedagogía, 429, 14-16.

VALLS, R., PRADOS, M. M. Y AGUILERA, A. (2014). El proyecto INCLUDED: estrategias para la inclusión y la cohesión social en Europa desde la educación. Investigación en la Escuela, 82, 31-44.

VIEIRA, L. (2010). Voluntariado en la escuela: un estudio de casos dentro del proyecto Comunidades de Aprendizaje. Tesis Doctoral. Recuperado de http://www.tdx.cat/bitstream/handle/10803/21615/01.LVP_TESIS.pdf?Sequence=1

VYGOTSKI, L. S. (1979). El desarrollo de los procesos psicológicos superiores. Barcelona: Crítica, 1989 ( $2^{a}$ edición). 


\section{Correspondencia con los autores}

María del Mar PRADOS GALLARDO

Departamento de Psicología Evolutiva y de la Educación

Facultad de Ciencias de la Educación, Universidad de Sevilla.

C/ Pirotecnia s/n

41013 Sevilla

e-mail: marprados@us.es

María-Teresa GÓMEZ DEL CASTILLO

Departamento de Didáctica y Organización Escolar

Facultad de Ciencias de la Educación, Universidad de Sevilla.

C/ Pirotecnia s/n

41013 de Sevilla

e-mail: mgomezdelcastillo@us.es

Antonio AGUILERA JIMÉNEZ

Departamento de Psicología Evolutiva y de la Educación

Facultad de Psicología, Universidad de Sevilla.

C/ Camilo José Cela s/n

41018 Sevilla

e-mail: aguijim@us.es 